\title{
Article
}

\section{Nucleation and Growth of Covalent Organic Frameworks from Solution: The Example of COF-5}

\author{
Haoyuan Li, Anton D. Chavez, Huifang Li, Hong Li, William R. Dichtel, and Jean-Luc Bredas
}

J. Am. Chem. Soc., Just Accepted Manuscript • DOI: 10.1021/jacs.7b09169 • Publication Date (Web): 23 Oct 2017

Downloaded from http://pubs.acs.org on October 29, 2017

\section{Just Accepted}

"Just Accepted" manuscripts have been peer-reviewed and accepted for publication. They are posted online prior to technical editing, formatting for publication and author proofing. The American Chemical Society provides "Just Accepted" as a free service to the research community to expedite the dissemination of scientific material as soon as possible after acceptance. "Just Accepted" manuscripts appear in full in PDF format accompanied by an HTML abstract. "Just Accepted" manuscripts have been fully peer reviewed, but should not be considered the official version of record. They are accessible to all readers and citable by the Digital Object Identifier (DOI®). "Just Accepted" is an optional service offered to authors. Therefore, the "Just Accepted" Web site may not include all articles that will be published in the journal. After a manuscript is technically edited and formatted, it will be removed from the "Just Accepted" Web site and published as an ASAP article. Note that technical editing may introduce minor changes to the manuscript text and/or graphics which could affect content, and all legal disclaimers and ethical guidelines that apply to the journal pertain. ACS cannot be held responsible for errors or consequences arising from the use of information contained in these "Just Accepted" manuscripts. 


\section{Frameworks from Solution: The Example of COF-5}

Haoyuan Li, ${ }^{1,2}$ Anton D. Chavez, ${ }^{3,4}$ Huifang Li, ${ }^{2}$ Hong Li, ${ }^{1,2}$ William R. Dichtel, ${ }^{3 *}$ and Jean-Luc Bredas $^{1,2 *}$

${ }^{1}$ School of Chemistry and Biochemistry, Center for Organic Photonics and Electronics (COPE), Georgia Institute of Technology, Atlanta, Georgia 30332-0400, United States.

${ }^{2}$ Laboratory for Computational and Theoretical Chemistry of Advanced Materials, Physical Science and Engineering Division, King Abdullah University of Science and Technology, Thuwal 23955-6900, Kingdom of Saudi Arabia

${ }^{3}$ Department of Chemistry, Northwestern University, Evanston, Illinois 60208, United States

${ }^{4}$ Department of Chemistry and Chemical Biology, Baker Laboratory, Cornell University, Ithaca, New York 14853, United States

"Emails: jean-luc.bredas@chemistry.gatech.edu; wdichtel@northwestern.edu

KEYWORDS.

covalent organic frameworks (COFs), nonclassical crystallization, crystallization by particle attachment, kinetic Monte Carlo simulation 


\begin{abstract}
The preparation of two-dimensional covalent organic frameworks (2D COFs) with large crystalline domains and controlled morphology is necessary for realizing the full potential of their atomically precise structures and uniform, tailorable porosity. Currently 2D COF syntheses are developed empirically, and most materials are isolated as insoluble and unprocessable powders with typical crystalline domain sizes smaller than $50 \mathrm{~nm}$. Little is known about their nucleation and growth processes, which involve a combination of covalent bond formation, degenerate exchange, and non-covalent stacking processes. A deeper understanding of the chemical processes that lead to COF polymerization and crystallization is key to achieving improved materials quality and control. Here, we report a kinetic Monte Carlo (KMC) model that describes the formation of a prototypical boronate-ester linked 2D COF known as COF-5 from its 2,3,6,7,10,11-hexahydroxytriphenylene and 1,4-phenylene bis(boronic acid) monomers in solution. The key rate parameters for the KMC model were derived from experimental measurements when possible and complemented with reaction pathway analyses, molecular dynamics simulations, and binding free-energy calculations. The essential features of experimentally measured COF-5 growth kinetics are reproduced well by the KMC simulations. In particular, the simulations successfully captured a nucleation process followed by a subsequent growth process. The nucleating species are found to be multi-layer structures that form through multiple pathways. During the growth of COF-5, extensions in the lateral (inplane) and vertical (stacking) directions are both seen to be linear with respect to time and are dominated by monomer addition and oligomer association, respectively. Finally, we show that the experimental observations of increased average crystallite size with the addition of water are
\end{abstract}


modeled accurately by the simulations. These results will inform the rational development of 2D COF polymerizations to control the rate of nucleation, thereby increasing their materials quality.

1

2

3

4

5

6

7

8

9

10

11

12

13

14

15

16

17

18

19

20

21

22

23

24

25

26

27

28

29

30

31

32

33

34

35

36

37

38

39

40

41

42

43

44

45

46

47

48

49

50

51

52

53

54

55

56

57

58

59

60 


\section{Introduction}

Covalent organic frameworks $(\mathrm{COFs})^{1-6}$ are porous polymer networks that predictably incorporate their monomers into periodic two-dimensional (2D) or three-dimensional (3D) topologies. 2D COFs in particular provide excellent structural versatility and tailorable few nmsize pores that suggest potential applications for catalysis ${ }^{7,8}$, optoelectronic devices ${ }^{9,10}$, electrical energy storage ${ }^{11,12}$, and nanofiltration membranes ${ }^{13}$, among others ${ }^{3,14-17}$. However, 2D COFs are typically isolated as insoluble and unprocessable powders with nanometer-scale in-plane crystalline domain sizes, which greatly limits their utility. If COFs are to be used to their fullest potential, enlarging their crystalline domains and gaining temporal and spatial control of nucleation and growth are necessary.

A prototypical boronate ester-linked 2D COF (COF-5, Figure 1), which is derived from the condensation of 2,3,6,7,10,11-hexahydroxytriphenylene (HHTP) and 1,4-phenylene bis(boronic acid) (PBBA), exhibits average in-plane domain sizes on the order of $20 \mathrm{~nm} .{ }^{18}$ These microcrystalline powder forms arise from uncontrolled nucleation and growth processes, both of which remain poorly understood and largely unstudied. The polymerization conditions used to prepare COF-5, as well as other similar materials, are typically determined empirically, often by varying experimental parameters in parallel and screening for crystalline precipitates using powder X-ray diffraction. This method of discovery is biased towards identifying conditions under which nucleation is rapid, and little information is obtained from experiments that fail to provide crystalline products. A robust understanding of the microscopic processes associated with the formation and growth of 2D COFs is required to improve their materials quality (e.g., to obtain larger crystallites and control their isolated form). Smith and Dichtel recently performed the first detailed kinetic study of COF-5 formation, ${ }^{18}$ in which they monitored the rate of COF-5 
precipitation after a few-minute induction period as a function of turbidity. Measuring this rate at different temperatures, concentrations, and in the presence of various additives provided activation energies and insight into the presence of both reversible and irreversible processes. However, turbidity measurements directly report on processes downstream of the initial polymerization and nucleation processes, which have yet to be probed experimentally. ${ }^{19,20}$ Therefore, detailed simulations as reported here can inform our understanding of the principles behind COF growth and ultimately contribute to robust, modular, and controlled methods to prepare 2D polymer networks.

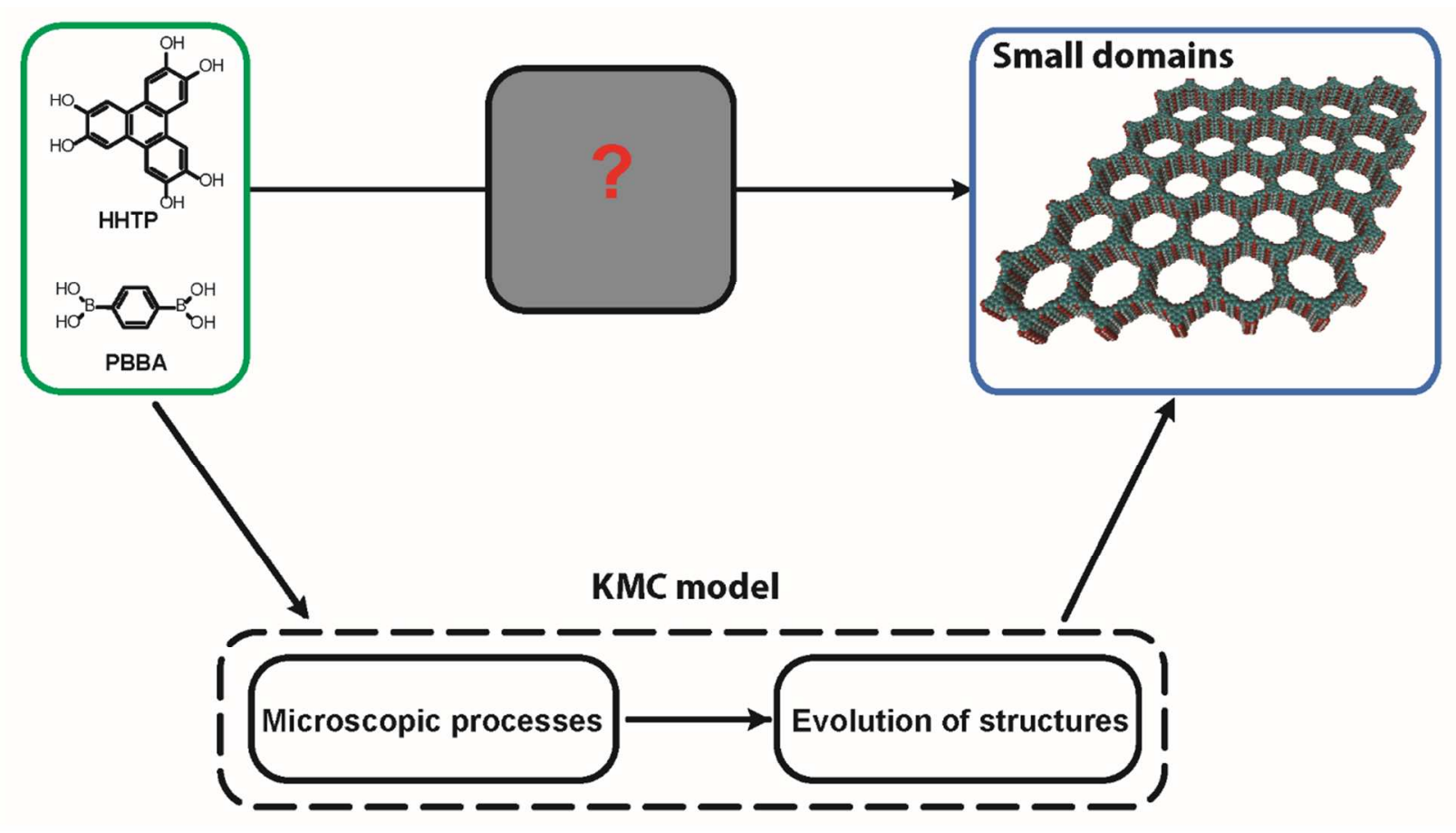

Figure 1. Illustration of the use of the KMC model to understand the crystallization of COF-5. 
Crystallization starts with nucleation followed by growth. ${ }^{21}$ The classical theories of crystallization, classical nucleation theory $(\mathrm{CNT})^{22}$ and classical crystal growth theory ${ }^{23-25}$, assume monomer-by-monomer addition. Although recent experimental findings show that many crystallizations do not strictly adhere to this assumption for nucleation ${ }^{26-33}$ and growth ${ }^{34-40}$, CNT is still widely used even when its assumptions are obviously violated. ${ }^{41}$ It remains challenging to derive a comprehensive and general theory to describe crystallization processes because of the diversity of crystallization processes and the difficulty in characterizing them. ${ }^{41,42}$ Nucleation is a dynamic process that occurs within nanoseconds at the nanometer scale, and often in a medium of complex composition. These factors make it hard to probe experimentally, particularly for 2D COFs, which undergo simultaneous polymerization and non-covalent assembly. ${ }^{31,41,43-46}$ Nucleation is also stochastic and rare, which complicates molecular dynamics (MD) simulations; crystallization simulations require long time scales and large sizes, particularly for complex processes such as crystallization from solution. ${ }^{41}$ Nucleation and growth have been considered to be governed by different mechanisms and modeled independently, ${ }^{24,47-50}$ despite the fact that they are intrinsically connected. Consistent descriptions of both the nucleation and growth in the same model is required for a more complete understanding of the crystallization process.

Here, we gain insight into the formation of COF-5 by developing a Kinetic Monte Carlo (KMC) approach that is able to describe COF-5 crystallization from monomers under experimental condition, a feature that has not been addressed to date. $\mathrm{KMC}$ is a general computational method used to describe the time evolution of a system. ${ }^{51,52}$ The system size and time scale accessible in a KMC simulation can be substantially larger and longer than those in MD simulations. KMC has been successfully applied to chemical reactions ${ }^{51-53}$, polymer growth ${ }^{54}$, charge transport ${ }^{55-57}$, predicting the kinetic factors for nucleation ${ }^{58,59}$, describing the growth of crystals ${ }^{60-64}$, and lateral 
growth of single-layer $2 \mathrm{D}$ materials ${ }^{65-68}$. Our $\mathrm{KMC}$ model reproduces key experimentally measured parameters of COF-5 growth, and provides new insights into the crystallization of COF-5. Importantly, the model simultaneously describes the nucleation and growth processes, with no predefined stages / pathways. By combining experimental rate data, reaction pathway analyses, MD simulations, and binding free-energy calculations, we are able to determine the rate parameters associated with the microscopic processes in the KMC model. We then use our $\mathrm{KMC}$ model as a platform to investigate the processes of nucleation and growth (both laterally and vertically) of the COF-5 crystal, providing the first structural insight into its nucleating species. The crystallization of COF-5 has a nonclassical feature, as there are multiple nucleation pathways and oligomers play an important role in the nucleation process and dominate vertical growth. We anticipate that these findings will lead to a more rational approach to $2 \mathrm{D} \mathrm{COF}$ synthesis, in contrast to current protocols in which small crystalline domain sizes originate from uncontrolled and rapid nucleation throughout the polymerization.

\section{The kinetic Monte Carlo model}

COF-5 is formed by the condensation of 2,3,6,7,10,11-hexahydroxytriphenylene and 1,4phenylenebis(boronic acid), traditionally in $4: 1$ dioxane:mesitylene (v/v) at elevated temperatures. ${ }^{1,18}$ The hexagonal sheets of COF-5 stack on each other in a quasi-eclipsed (cofacial) fashion with lateral domain sizes typically on the order of $20 \mathrm{~nm} .^{1,18}$

The KMC model developed to describe the crystallization of COF-5 from its monomers is based on the following assumptions: 
1. The key microscopic processes involved in the crystallization of COF-5 are bond formation, bond breakage, stacking, and destacking. These four microscopic processes are modeled as Markov processes, which means they are considered uncorrelated in time (for example, a bond formation process will not have any residual effect on a following stacking process). We note that microscopic processes such as the sliding of an oligomer on top of a larger 2D COF layer is expected to have only a modest influence on the crystallization process and are ignored in the KMC model. Both the MD simulations of Koo et al. ${ }^{69}$ and our own show that sliding of oligomers on COF-5 sheets is very limited (Figure S44), which validates their omission from the KMC model.

2. Eclipsed stacking is the only form of aggregation that is considered. This is a reasonable assumption considering the strong dispersion $(\pi-\pi)$ interactions between COF-5 sheets (described in the Sections 1 and 3 in the Supporting Information, SI) and the final structure of COF-5.

Figure 2 describes the conceptual framework of our KMC model.

In the KMC simulation, each monomer unit is considered as a node that can connect with other nodes. An interconnected node tree represents a specific oligomer. All oligomers are examined and identified according to their specific connection pattern. This approach, denoted as "node representation," is similar to the approach used to model polymer growth ${ }^{54,70-72}$. During the growth of COF-5, more complicated stacked structures can form, which require an explicit treatment of their molecular coordinates. To describe COF-5 crystals or smaller stacked structures, we consider eclipsed stacking using a predefined template according to the lattice structure and a specific occupation status in the template; this approach, similar to that used by 


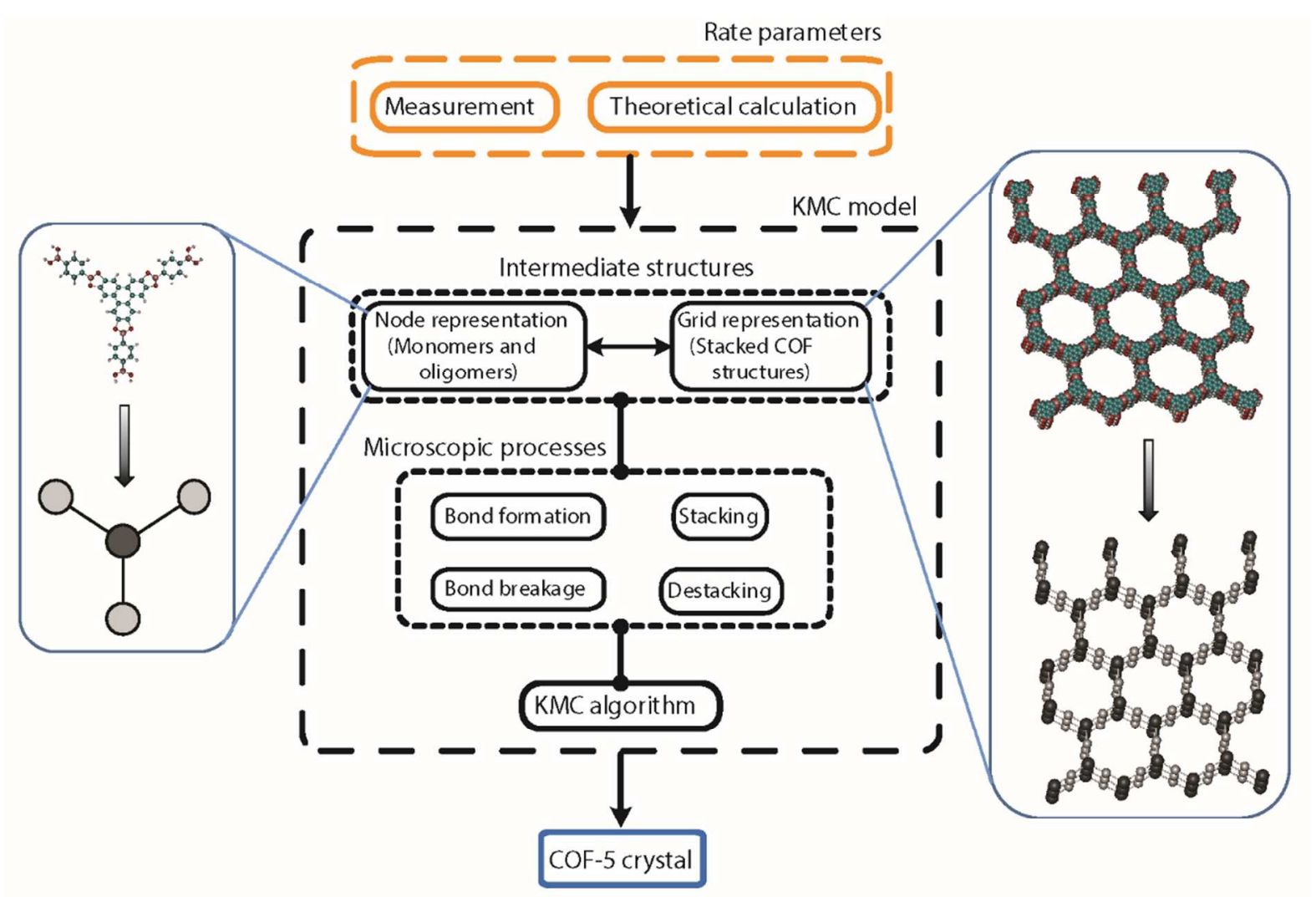

Figure 2. Flowchart of the KMC model developed to simulate the formation of COF-5 crystals from monomers (see text for details).

Bond formation occurs when one of the three reactive sites of HHTP connects with one of the two reactive sites of PBBA among monomers, oligomers, or larger stacked structures, releasing 
two $\mathrm{H}_{2} \mathrm{O}$ molecules. Conversely, bond breakage occurs when a connection between HHTP and PBBA is severed, consuming two $\mathrm{H}_{2} \mathrm{O}$ molecules. Since bond formation and breakage are dependent on the concentration of monomers, oligomers, aggregated structures, and $\mathrm{H}_{2} \mathrm{O}$, the KMC simulation is updated at every step to account for the current composition.

Stacking can occur between two oligomers, one oligomer and a stacked structure, or two stacked structures. The grid representation is used when two structures stack into a larger eclipsed structure (with any HHTP unit in one structure having equal chance to stack with any HHTP unit in the other structure at the interface). Destacking occurs when two adjacent layers in a COF structure are split into two new structures. When the products are multi-layered, the grid representation is used, otherwise the node representation is employed.

Once the size of a COF structure grows past a given threshold, it is considered as a precipitate, recorded, and removed out of the simulation. A threshold of 5000 monomer units is used, since the largest average diameter $(\sim 45 \mathrm{~nm}$, Figure S84) of the simulated COFs is then similar to that of materials obtained under optimal experimental conditions $\left(\sim 40 \mathrm{~nm}^{18}\right)$.

The random selection method $(\mathrm{RSM})^{53}$ is adopted in the KMC simulation, meaning that at every step all events can happen with probabilities that are proportional to their associated rates. The state resident time $(\tau)$ is expressed as:

$$
\tau=-\frac{\ln \left(\xi_{1}\right)}{\sum_{i} v_{i}}
$$

where $\xi_{1}$ is a random number uniformly distributed between 0 and 1 and $v_{i}$ is the rate of the microscopic process (event) $i$. At each step, event $j$ is selected to execute, via the condition: 


$$
\frac{\sum_{1}^{j-1} v_{i}}{\sum_{i} v_{i}}<\xi_{2} \leq \frac{\sum_{1}^{j} v_{i}}{\sum_{i} v_{i}}
$$

where $\xi_{2}$ is a random number uniformly distributed between 0 and 1.

Extreme optimization of the KMC code has allowed us to simulate systems with sizes of $\sim 10^{5}$ monomers in the time scale of hours. The rate parameters are estimated through a combination of experimental and theoretical measurements, which we detail in the Section $\mathbf{1}$ in the SI. While the solvent is not explicitly considered in the $\mathrm{KMC}$ model, its influence is considered in the evaluation of appropriate rate parameters.

\section{Results and discussion}

The KMC simulation qualitatively reproduces the experimental growth curve of COF-5 (Figure 3). Both curves contain an induction period followed by a linear production with time. The induction period suggests a pre-equilibrium process in the early stage of the crystallization process, while the existence of a linear regime suggests a steady nucleation rate.

We note that the induction period and the production rate from the KMC simulations quantitatively differ from the experimental results, which highlights the challenge in accurately calculating the rates involved in crystallization..$^{21,73}$ The simulated induction period $(\sim 10 \mathrm{~s}$ compared to $\sim 2 \mathrm{~min})$ is shorter and the simulated initial growth rate $(97.5 \%$ per min compared to $17.1 \%$ per $\min$ ) is faster. However, these differences are within one order of magnitude or less, which in the present context is generally considered to be small. ${ }^{21,41,74}$ Nevertheless, these 
differences likely arise because (i) turbidity measurements monitor aggregates of crystals in the scale of hundreds of nanometers or microns whereas the KMC simulation monitors all sizes of COF-5 crystals and (ii) the measured rate parameters were obtained from the reactions of 4-tertbutylcatechol (TCAT) with PBBA while our reaction pathway analyses suggest that the reaction energy barrier for HHTP and PBBA can be larger (see Section 3 in SI), which will influence the KMC results. As discussed below, the nucleation process is highly influenced by the rate of bond formation. 

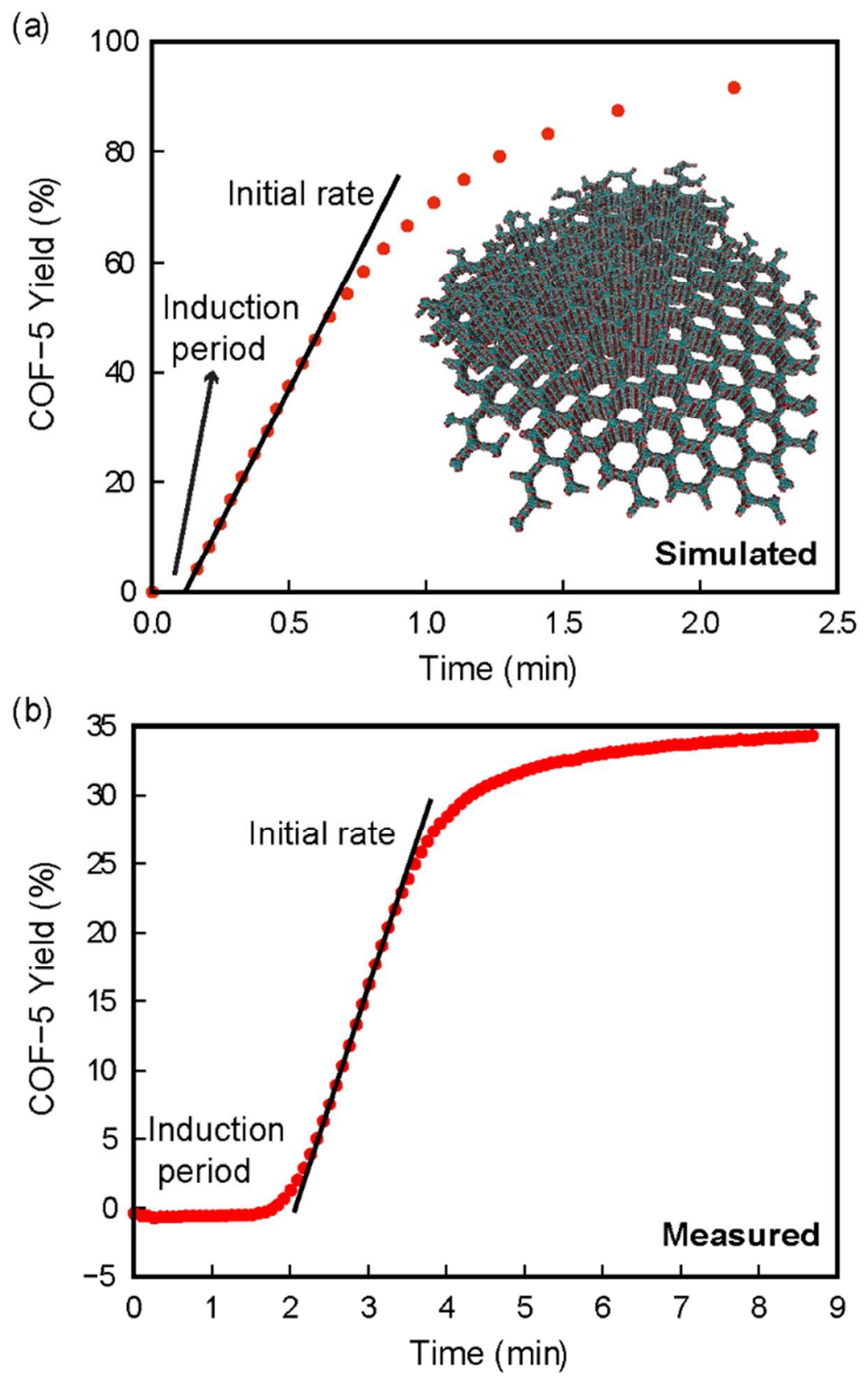

Figure 3. (a) Simulated and (b) measured COF-5 yields with time. Inset in (a) shows a COF-5 crystal from the KMC simulation. Black lines mark the linear production regime. In the turbidity measurement, the COF-5 yield is directly proportional to turbidity up until $c a .27 \%{ }^{18}$ The initial concentrations of HHTP and PBBA are $8 \mathrm{mM}$ and $12 \mathrm{mM}$, respectively. 


\subsection{Nucleation}

To understand the structure of the nuclei and the nucleation process, we monitored all the generated intermediate structures in the KMC simulations once they have reached a certain number of monomer units $(N)$. As seen in Figure 4a, small structures (e.g., with 15 monomer units) have very low probabilities of finally growing into COF-5 crystals, with the majority falling back apart. However, larger structures (with 30 or more monomer units) nearly always grow into a COF-5 crystal.

This suggests the existence of a nucleation process involving the evolution of these small boronate-ester linked structures. If we neglect the structural differences and assume that there is only one nucleation pathway, the partial free-energy profile can be estimated (see Figures S85S86 in SI). When relating to classical nucleation theory ${ }^{22}$, the critical nucleus size is around 25 monomer units. We find that the nuclei are multi-layer structures (Figures $\mathbf{4 b}$ and $\mathbf{S 8 7}$ ) and that the nucleation process is the formation of stable stacked structures that allow further growth before they dissociate.

Stacking between oligomers is a fast process with a negligible energy barrier (see Section 4.3 in SI), frequently resulting in multi-layered structures. The rate of the reverse process, destacking, is highly dependent on the size of the oligomers. It is estimated that a two-layer structure with an interfacial area of seven monomer units can subsist for over $1 \mathrm{~s}$ before destacking, and larger structures remain stable for much longer (Figure S82). This is in agreement with our recent report that macrocycles resembling COF-5 (consisting of 12 monomer units) spontaneously stack into nanotubes. ${ }^{75}$ Larger structures have larger lateral sizes (average number of monomer units 
per layer, as shown in Figure 4b), and thus become intrinsically more stable, which explains the results illustrated in Figure 4a.

When considering different intermediate structures $(N=15), 97.5 \%$ of them are three-layered while only $2.5 \%$ are two-layered (Figure 4c). However, only $0.01 \%$ of the three-layer structures grow into COF-5 crystals, while a much higher ratio of $2.6 \%$ of the two-layer structures do the same. As a result, if we only consider the structures that eventually grow into COF-5 crystals (denoted as "success" in Figure 4c), the majority of them $(\sim 80 \%)$ are twolayered. This can be understood by the fact that two-layer structures can have a larger interfacial area and thus are more stable. Overall, these different structures represent two nucleation pathways for how differently sized oligomers can stack and grow. 

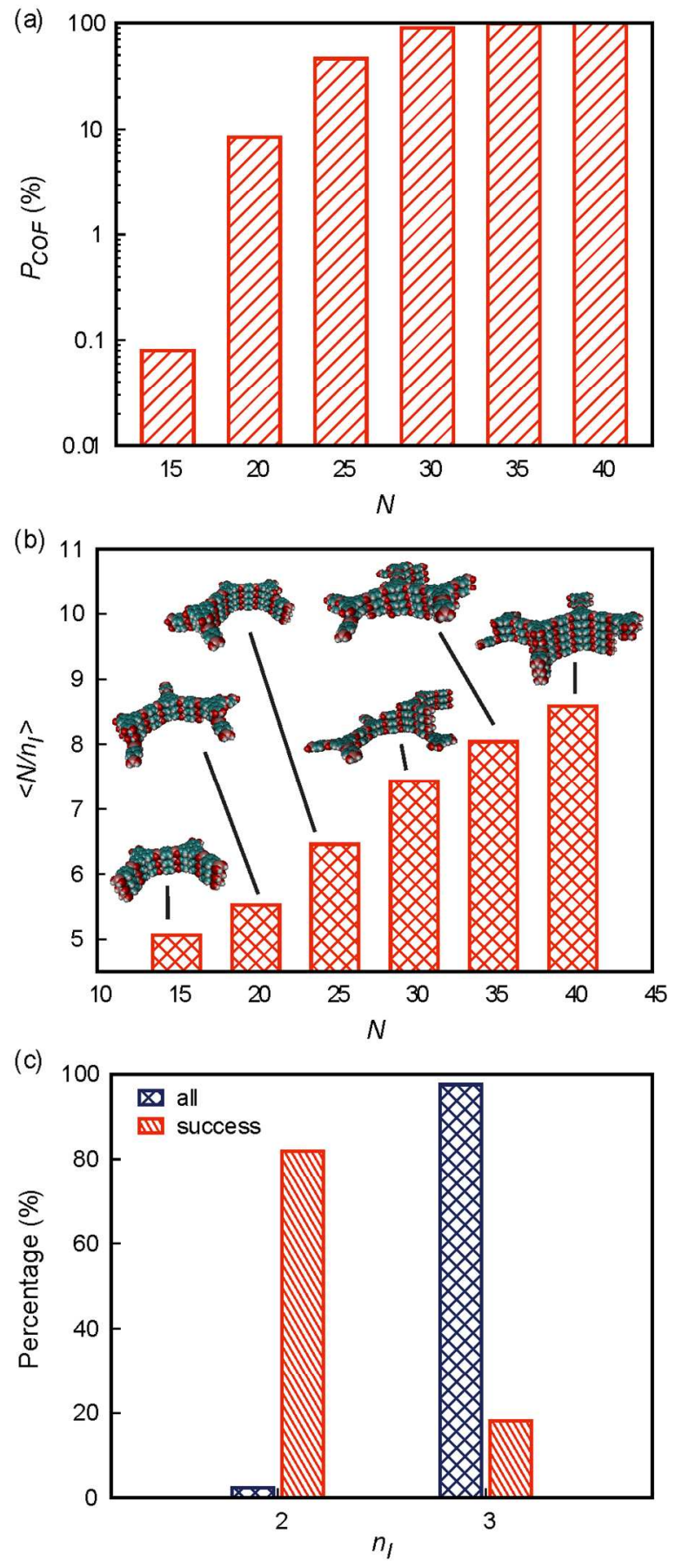

Figure 4. (a) Probabilities of different-size intermediate structures to grow into COF-5 crystals $\left(P_{C O F}\right)$. (b) Average number of monomer units per layer for the intermediate structures; insets show representative structures at different sizes. (c) Percentages of intermediate structures $(N=15)$ belonging to different numbers of layers; "all" denotes the percentage of all the monitored structures with those numbers of layers while "success" denotes the percentage of those that finally grow into COF-5 crystals. 
The nucleation of COF-5 is a multistage process, as depicted in Figure 5. The first stage is the reaction of monomers to form small oligomers, which can only happen at elevated temperatures due to the relatively high reaction barriers. The time scale of this initial stage is influenced by the bond formation rate constant and is strongly correlated with the induction time seen in Figure 2a. Reducing the bond formation rate constant increases the induction time (Figure S88). These oligomers then follow two different nucleation pathways (Figure 5), corresponding to the differences highlighted in Figure 4c. In one pathway, these small oligomers stack into larger structures. While the majority destack back into oligomers, a small population can grow laterally. This produces structures with larger interfacial area between layers, increasing stability, and allows for further growth. In the second nucleation pathway, small oligomers gradually continue to grow laterally, forming large oligomers that can subsequently stack and produce stable nuclei that further grow. While the results in Figure 4c (red bars) suggest that most nuclei come from the second pathway, these two pathways are not mutually exclusive. When the first nucleation pathway fails, the generated oligomers are able to participate in the second nucleation pathway (green dashed lines in Figure 5b). The early stages of nucleation resemble a step-growth polymerization $^{76}$, but with a major difference that oligomers stack instead of forming bonds. 
(a)

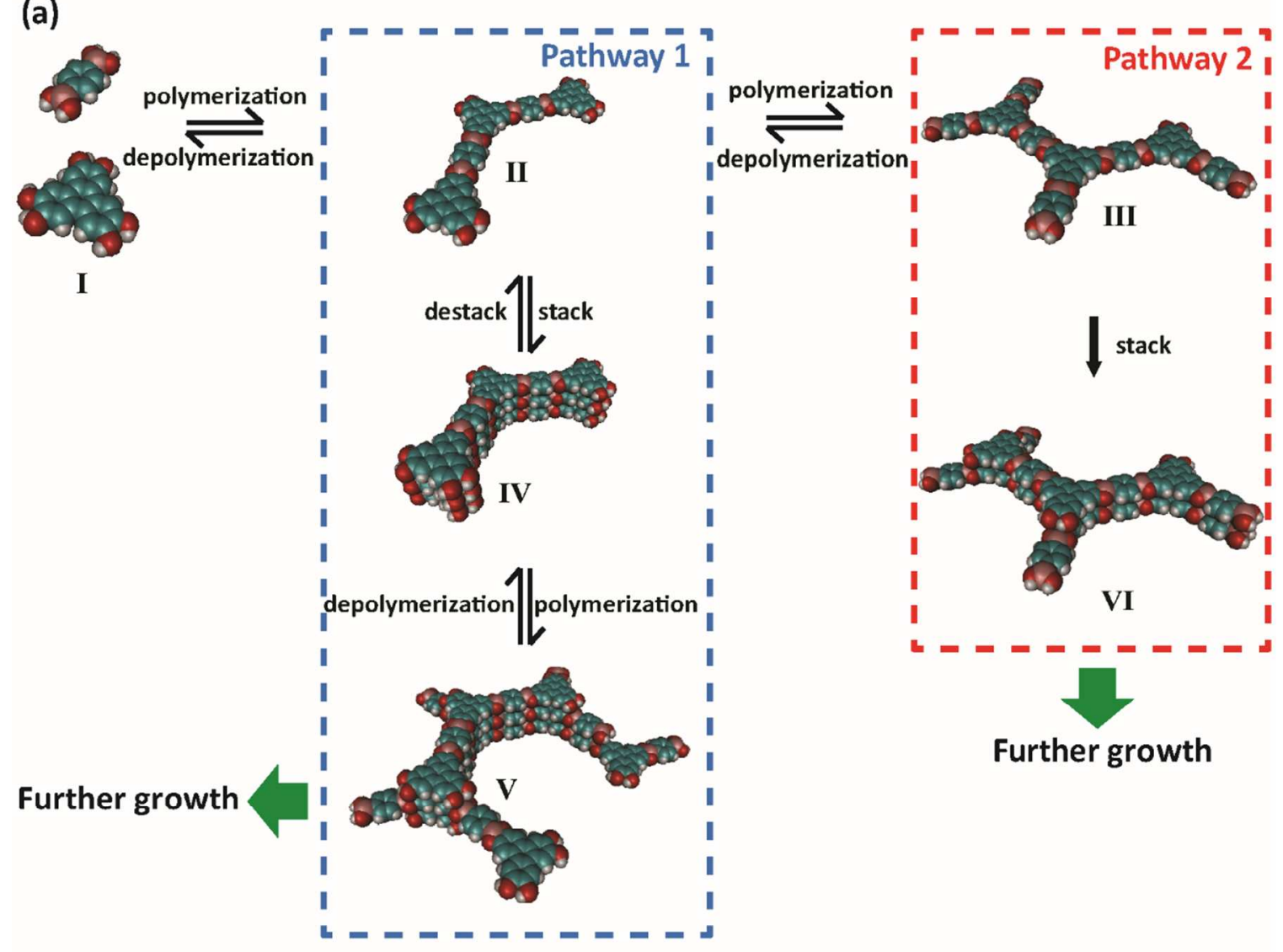

(b)

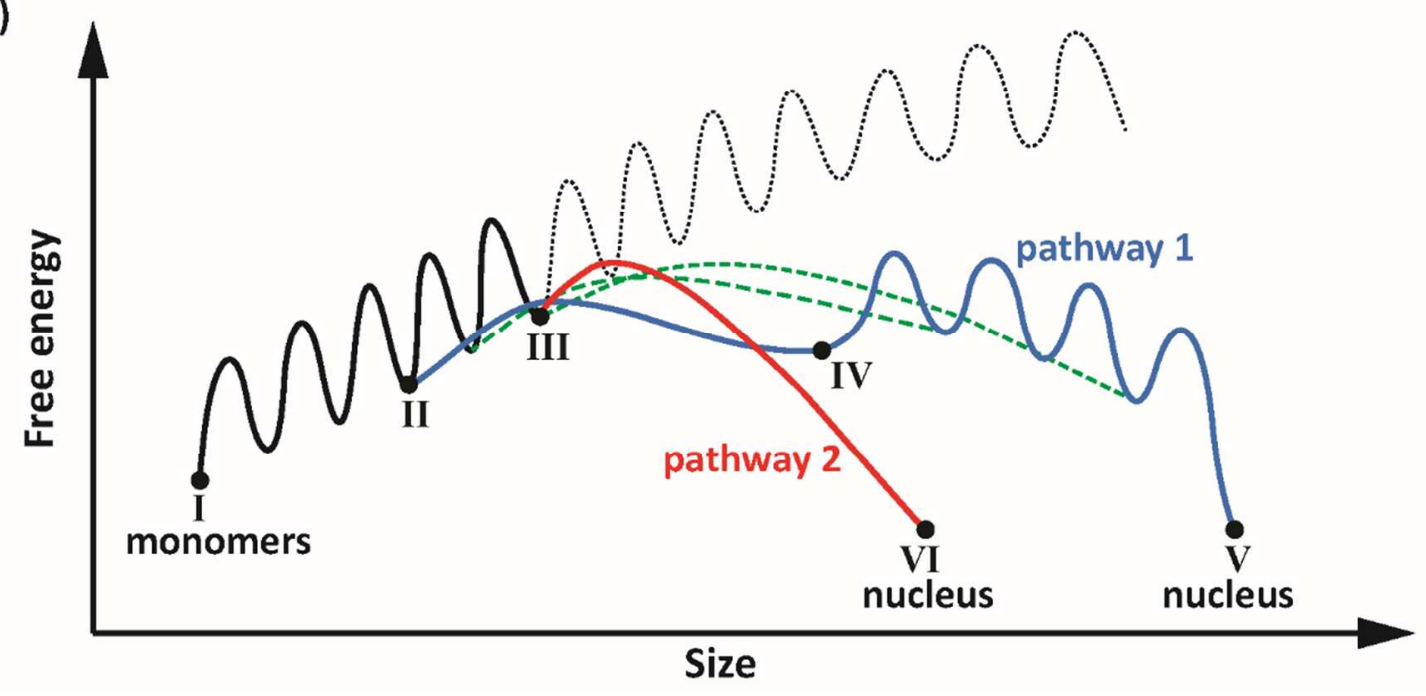

Figure 5. (a) Two observed nucleation pathways for COF-5. (b) Proposed energy diagram for the nucleation of COF-5 (black and dotted lines represent bond formation and breakage; blue and red lines represent the first and second nucleation pathways, respectively; the green dashed lines represent the exchange of species between the two pathways). 


\subsection{Growth}

After a nucleus is formed, it continues to grow into a COF-5 crystal. Monitoring the formation of a single crystal shows that both the averaged lateral diameter among layers $\left(d_{\text {ave }}\right)$ and the height (h) increase approximately linearly with time (Figure 6) with final sizes comparable to the dimensions of the average crystallite size inferred for a typical COF-5 sample. The linear growth suggests a chain-growth-like mechanism ${ }^{76}$, whereas a step-growth polymerization would lead to a non-linear growth dynamics (see Figure S89).

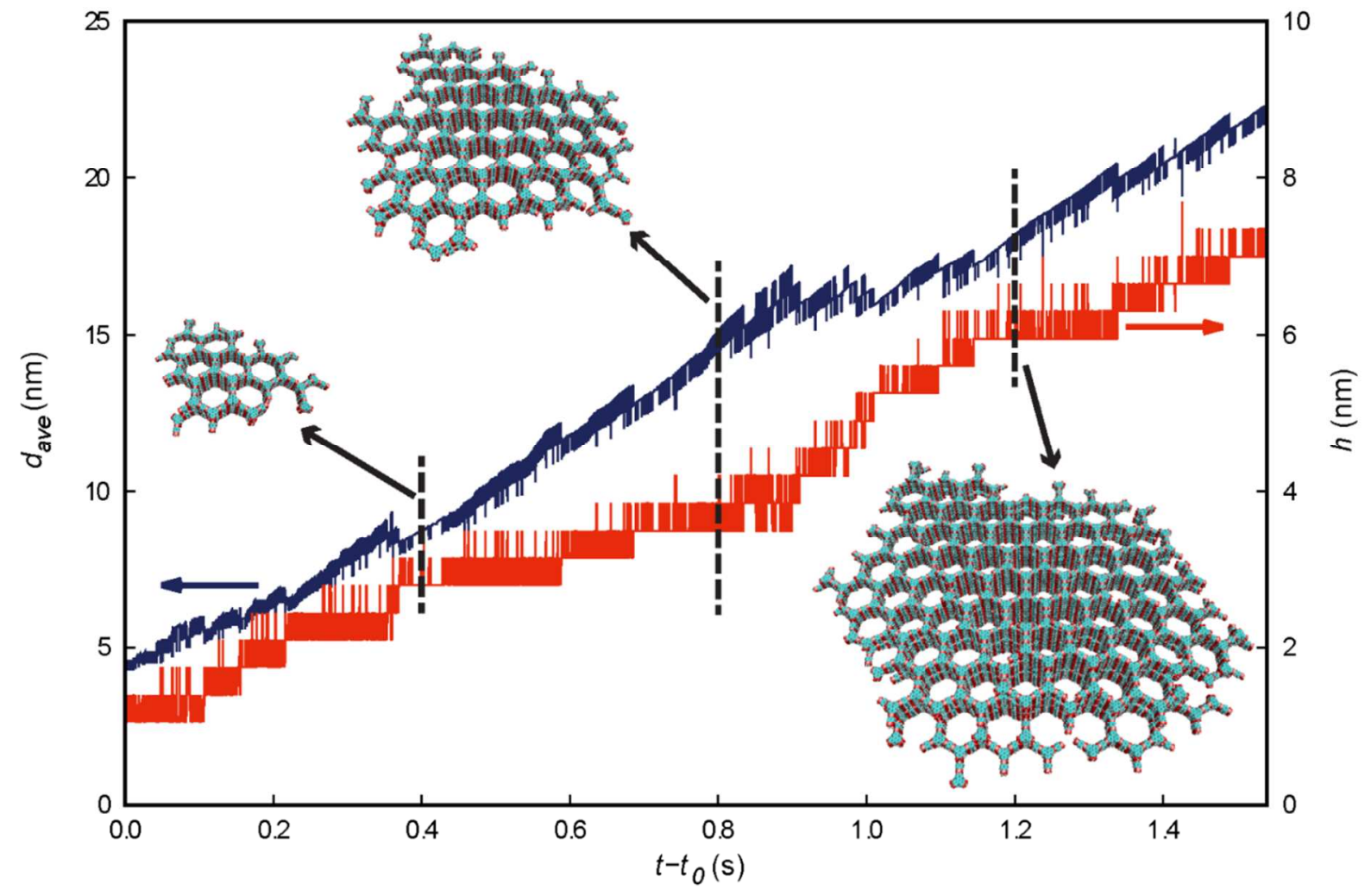

Figure 6. Averaged diameter among layers $\left(d_{\text {ave }}\right)$ and height $(h)$ of the COF-5 crystal during its growth. Insets show the structures at different times. $t_{0}$ denotes the time when the crystal started to be monitored, which corresponds to a nucleus with a total number of 40 monomer units. 
Analysis of all the molecular species that were incorporated in the COF-5 crystals reveals that they are mostly monomers that formed bonds with COF-5 during the growth (Figure 7). This is understandable since monomers are present in larger numbers compared to larger oligomers (Figure S90). However, the contribution of oligomers (up to 4 monomer units) is not negligible since they are larger in size than monomers; limiting the maximum size of the oligomers that can bond with the COF-5 crystal leads to smaller crystal diameters (Figure S91). These results suggest that lateral growth (increase in $d_{\text {ave }}$ ) occurs through bond formation with monomers and small oligomers.

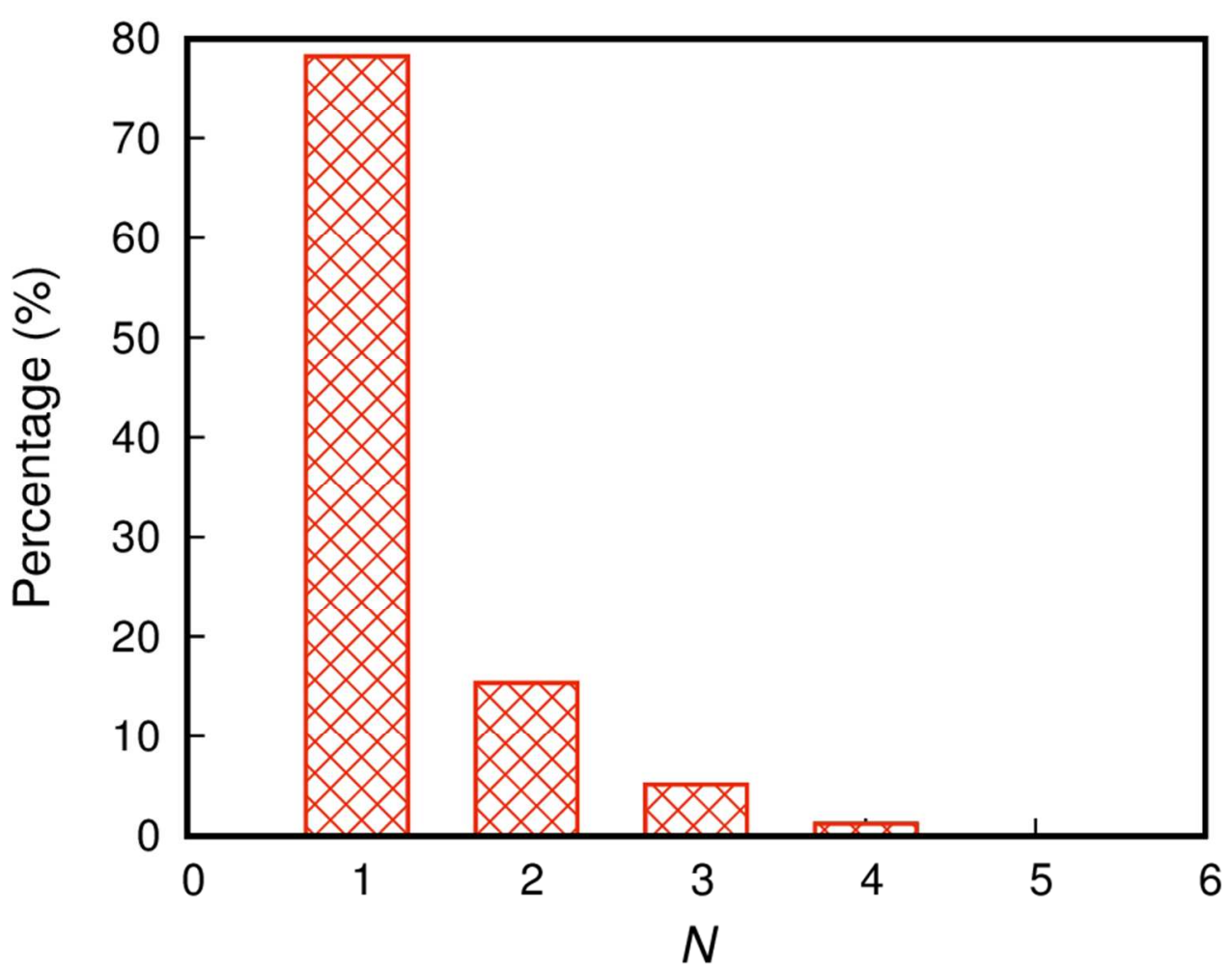

Figure 7. Percentages of monomers and oligomers with $N$ monomer units among all particles that bond with COF-5 crystals during their growth. 
The growth of a crystal in 3D space requires increasing the number of atomic / molecular layers. The growth of a new layer starts with the birth of a 2D nucleus on top of an old layer, which has been considered to form via monomer-by-monomer addition on top of this old layer or through dislocations. $^{23,25}$ In the case of COF-5 crystals, monomers are found not to stack well on COF sheets (Figure S45), and a new 2D nucleus can result from the stacking between the crystal and thermodynamically metastable oligomers present in the system, which can also include subsequent lateral growth of the stacked oligomer that further stabilizes the nucleus. For example, an oligomer of 6 or more monomer units stacks well on existing COF-5 sheets and become a 2D nucleus for a new layer; an oligomer of 5 monomer units forms a less stable stacked structure with COF-5 sheets, but can become stable if it bonds with a monomer before destacking. Our MD and KMC simulations indicate that the vertical (normal) growth, corresponding to the formation of new layers, is dominated by oligomers. Finally, the addition of water to the reaction mixture has previously been shown to increase the crystallite size of COF5. ${ }^{18}$ This feature is successfully reproduced by the KMC simulations, see Figure 8. We hypothesize that higher concentrations of $\mathrm{H}_{2} \mathrm{O}$ shift the equilibrium from large oligomers towards monomers, thereby favoring lateral growth over vertical growth. 


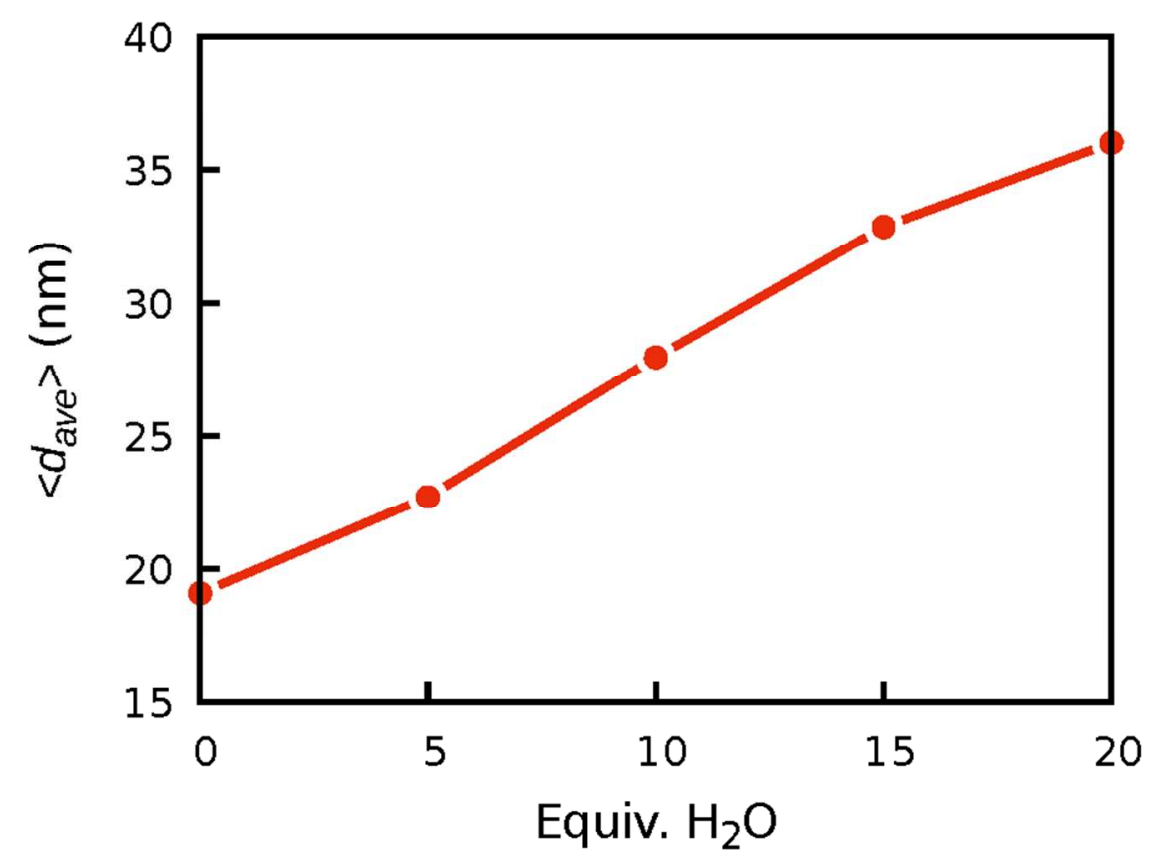

Figure 8. Average diameter (among layers) of COF-5 crystals at different initial $\mathrm{H}_{2} \mathrm{O}$ concentrations.

\subsection{Nonclassical crystallization of COF-5}

Traditionally, crystallization is considered to follow the classical theory based on monomer-bymonomer addition. However, it has recently been shown that nonclassical crystallization occurs in many systems: ${ }^{21,48,50,77-82}$ Nucleation can have a two-step mechanism that differs from that described by classical nucleation theory; ${ }^{77,83-87}$ particles larger than monomers / ions can participate in the growth of crystals $;^{30,39,40}$ an oriented attachment (OA) mechanism has also been identified, in which nanocrystals coalesce to form the bulk crystal. ${ }^{34,36-38,88}$ The term "crystallization by particle attachment (CPA)" is now used to denote crystallization processes that are dominated by particles other than monomers / ions. ${ }^{42}$ Our results demonstrate that the 
crystallization of COF-5 is nonclassical and show strong CPA features: Oligomers are key precursors for nucleation, are major contributors to vertical growth, and contribute slightly to lateral growth.

Compared with other CPA mechanisms, the crystallization of COF-5 has its own specificities: Nucleation involves multiple thermodynamically metastable species (oligomers, stacked structures), lateral growth and vertical growth have different mechanisms, and the (classical) monomer-by-monomer addition mechanism is still present and dominates the lateral growth of COF-5 crystals. These results highlight the diversity of the crystallization process among different materials. $^{41}$

\section{Conclusions}

To summarize, we have developed a kinetic Monte Carlo (KMC) model for the crystallization of COF-5 from solution, which reproduces experimental results well. Experimental and theoretical approaches have been combined to estimate the rate parameters associated with the microscopic processes. Based on our KMC simulations, we are able to provide a comprehensive understanding of the nucleation and growth of COF-5.

The nucleation process has several stages and results in multi-layer structures. The formation of small oligomers is the first stage and influences the length of the induction period. Two nucleation pathways have been identified: (i) lateral growth of small stacked structures; and (ii) stacking between large oligomers. During the growth of COF-5, both diameter and height increase linearly with time. Monomers dominate the lateral growth, while vertical growth is 
primarily due to oligomers stacking. Adding $\mathrm{H}_{2} \mathrm{O}$ shifts the equilibrium away from large oligomers, favoring lateral growth over vertical growth and resulting in COF-5 crystals with larger diameters. These results show that the crystallization of COF-5 is reminiscent of crystallization by particle attachment (CPA), since oligomers play an important role in the nucleation process and dominate vertical growth.

Overall, we have demonstrated the feasibility of a multi-pronged approach to model the complex crystallization process of COFs. Importantly, both nucleation and growth are shown to be consistently described within the same framework, two processes that are classically modeled separately. We believe that our results will lead to informed 2D COF syntheses where both nucleation and growth will be controlled yielding higher quality materials.

\section{ASSOCIATED CONTENT}

Supporting Information. Details about the rate parameters in the KMC model, the experiments, reaction pathway analyses, MD simulations, binding free-energy calculations, and additional results from the KMC simulations. This material is available free of charge via the Internet at http://pubs.acs.org.

\section{AUTHOR INFORMATION}

Corresponding Author

*E-mail: jean-luc.bredas@chemistry.gatech.edu; wdichtel@northwestern.edu. 


\title{
ACKNOWLEDGMENTS
}

\author{
We would like to thank Dr. Brian J. Smith (Cornell) and Dr. Zhen Cao (KAUST) for helpful \\ discussions. The work at KAUST was supported by internal funding from King Abdullah \\ University of Science and Technology; we are grateful to the KAUST IT Research Computing \\ Team and Supercomputing Laboratory for providing outstanding assistance as well as \\ computational and storage resources. A.D.C. was supported by a National Defense Science and \\ Engineering Graduate Fellowship. This work made use of the IMSERC at Northwestern \\ University, which has received support from the Soft and Hybrid Nanotechnology Experimental \\ (SHyNE) Resource (NSF NNCI-1542205), the State of Illinois, and International Institute for \\ Nanotechnology (IIN). The collaborative work at Northwestern and Georgia Tech was supported \\ by the Army Research Office under the MURI Center for Advanced Two-dimensional Organic \\ Networks, CATON, under Award No. W911NF-15-1-0447, and under Award No. W911NF-17- \\ 1-0339 to Georgia Tech.
}




\section{REFERENCES}

(1) Cote, A. P.; Benin, A. I.; Ockwig, N. W.; O'Keeffe, M.; Matzger, A. J.; Yaghi, O. M. Science 2005, 310, 1166.

(2) Colson, J. W.; Dichtel, W. R. Nature Chem. 2013, 5, 453.

(3) Ding, S. Y.; Wang, W. Chem. Soc. Rev. 2013, 42, 548.

(4) Huang, N.; Wang, P.; Jiang, D. L. Nat. Rev. Mater. 2016, 1, 16068.

(5) DeBlase, C. R.; Dichtel, W. R. Macromolecules 2016, 49, 5297.

(6) Bisbey, R. P.; Dichtel, W. R. ACS Cent. Sci. 2017, 3, 533.

(7) Lin, S.; Diercks, C. S.; Zhang, Y. B.; Kornienko, N.; Nichols, E. M.; Zhao, Y. B.; Paris, A. R.; Kim, D.; Yang, P.; Yaghi, O. M.; Chang, C. J. Science 2015, 349, 1208.

(8) Xu, H.; Gao, J.; Jiang, D. L. Nature Chem. 2015, 7, 905.

(9) Guo, J.; Xu, Y. H.; Jin, S. B.; Chen, L.; Kaji, T.; Honsho, Y.; Addicoat, M. A.; Kim, J.; Saeki, A.; Ihee, H.; Seki, S.; Irle, S.; Hiramoto, M.; Gao, J.; Jiang, D. L. Nat. Commun. 2013, 4, 2736.

(10) Jin, S. B.; Ding, X. S.; Feng, X.; Supur, M.; Furukawa, K.; Takahashi, S.; Addicoat, M.; El-Khouly, M. E.; Nakamura, T.; Irle, S.; Fukuzumi, S.; Nagai, A.; Jiang, D. L. Angew. Chem., Int. Ed. Engl. 2013, 52, 2017.

(11) DeBlase, C. R.; Silberstein, K. E.; Truong, T. T.; Abruna, H. D.; Dichtel, W. R. J. Am. Chem. Soc. 2013, 135, 16821.

(12) Mulzer, C. R.; Shen, L. X.; Bisbey, R. P.; McKone, J. R.; Zhang, N.; Abruna, H. D.; Dichtel, W. R. ACS Cent. Sci. 2016, 2, 667.

(13) Lin, L.-C.; Choi, J.; Grossman, J. C. Chem. Commun. 2015, 51, 14921.

(14) Gourdon, A. Angew. Chem., Int. Ed. Engl. 2008, 47, 6950.

(15) Feng, X.; Ding, X. S.; Jiang, D. L. Chem. Soc. Rev. 2012, 41, 6010.

(16) Wu, D. C.; Xu, F.; Sun, B.; Fu, R. W.; He, H. K.; Matyjaszewski, K. Chem. Rev. 2012, $112,3959$.

(17) Xiang, Z. H.; Cao, D. P.; Dai, L. M. Polym Chem-Uk 2015, 6, 1896.

(18) Smith, B. J.; Dichtel, W. R. J. Am. Chem. Soc. 2014, 136, 8783.

(19) De Yoreo, J.; Whitelam, S. MRS bull. 2016, 41, 357.

(20) Rimer, J. D.; Tsapatsis, M. MRS bull. 2016, 41, 393.

(21) Erdemir, D.; Lee, A. Y.; Myerson, A. S. Acc. Chem. Res. 2009, 42, 621.

(22) Nanev, C. N. Handbook of Crystal Growth (Second Edition); Elsevier: Boston, 2015.

(23) Burton, W. K.; Cabrera, N.; Frank, F. C. Philos T R Soc S-A 1951, 243, 299.

(24) Teng, H. H. Elements 2013, 9, 189.

(25) Woodruff, D. P. Philos. Transact. A Math. Phys. Eng. Sci. 2015, 373, 20140230.

(26) Gebauer, D.; Volkel, A.; Colfen, H. Science 2008, 322, 1819.

(27) Pouget, E. M.; Bomans, P. H. H.; Goos, J. A. C. M.; Frederik, P. M.; de With, G.; Sommerdijk, N. A. J. M. Science 2009, 323, 1455.

(28) Van Driessche, A. E. S.; Benning, L. G.; Rodriguez-Blanco, J. D.; Ossorio, M.; Bots, P.; Garcia-Ruiz, J. M. Science 2012, 336, 69.

(29) Habraken, W. J. E. M.; Tao, J. H.; Brylka, L. J.; Friedrich, H.; Bertinetti, L.; Schenk, A. S.; Verch, A.; Dmitrovic, V.; Bomans, P. H. H.; Frederik, P. M.; Laven, J.; van der Schoot, P.; Aichmayer, B.; de With, G.; DeYoreo, J. J.; Sommerdijk, N. A. J. M. Nat. Commun. 2013, 4, 1507. 
(30) Baumgartner, J.; Dey, A.; Bomans, P. H. H.; Le Coadou, C.; Fratzl, P.; Sommerdijk, N. A. J. M.; Faivre, D. Nat. Mater. 2013, 12, 310.

(31) Agarwal, V.; Peters, B. Advances in Chemical Physics: Volume 155; John Wiley \& Sons, Inc.: New York, 2014.

(32) Peng, Y.; Wang, F.; Wang, Z. R.; Alsayed, A. M.; Zhang, Z. X.; Yodh, A. G.; Han, Y. L. Nat. Mater. 2015, 14, 101.

(33) Myerson, A. S.; Trout, B. L. Science 2013, 341, 855.

(34) Penn, R. L.; Banfield, J. F. Science 1998, 281, 969.

(35) Huang, F.; Zhang, H. Z.; Banfield, J. F. Nano Lett. 2003, 3, 373.

(36) Yuwono, V. M.; Burrows, N. D.; Soltis, J. A.; Penn, R. L. J. Am. Chem. Soc. 2010, 132, 2163.

(37) Liao, H. G.; Cui, L. K.; Whitelam, S.; Zheng, H. M. Science 2012, 336, 1011.

(38) Li, D. S.; Nielsen, M. H.; Lee, J. R. I.; Frandsen, C.; Banfield, J. F.; De Yoreo, J. J. Science 2012, 336, 1014.

(39) Lupulescu, A. I.; Rimer, J. D. Science 2014, 344, 729.

(40) Jiang, Y.; Kellermeier, M.; Gebauer, D.; Lu, Z.; Rosenberg, R.; Moise, A.; Przybylski, M.; Cölfen, H. Nat. Commun. 2017, 8, 15933.

(41) Sosso, G. C.; Chen, J.; Cox, S. J.; Fitzner, M.; Pedevilla, P.; Zen, A.; Michaelides, A. Chem. Rev. 2016, 116, 7078.

(42) De Yoreo, J. J.; Gilbert, P. U. P. A.; Sommerdijk, N. A. J. M.; Penn, R. L.; Whitelam, S.; Joester, D.; Zhang, H. Z.; Rimer, J. D.; Navrotsky, A.; Banfield, J. F.; Wallace, A. F.; Michel, F. M.; Meldrum, F. C.; Colfen, H.; Dove, P. M. Science 2015, 349, aaa6760.

(43) Anwar, J.; Zahn, D. Angew. Chem., Int. Ed. Engl. 2011, 50, 1996.

(44) Yi, P.; Rutledge, G. C. Annu. Rev. Chem. Biomol. 2012, 3, 157.

(45) Davey, R. J.; Schroeder, S. L. M.; ter Horst, J. H. Angew. Chem., Int. Ed. 2013, 52, 2166.

(46) Xu, X. F.; Ting, C. L.; Kusaka, I.; Wang, Z. G. Annu. Rev. Phys. Chem. 2014, 65, 449.

(47) Pan, W. C.; Kolomeisky, A. B.; Vekilov, P. G. J. Chem. Phys. 2005, 122, 174905.

(48) Burrows, N. D.; Yuwono, V. M.; Penn, R. L. MRS bull. 2010, 35, 133.

(49) Hu, Q.; Nielsen, M. H.; Freeman, C. L.; Hamm, L. M.; Tao, J.; Lee, J. R. I.; Han, T. Y. J.; Becker, U.; Harding, J. H.; Dove, P. M.; De Yoreo, J. J. Faraday Discuss. 2012, 159, 509.

(50) Linnikov, O. D. Russ. Chem. Rev. 2014, 83, 343.

(51) Gillespie, D. T. J. Comput. Phys. 1976, 22, 403.

(52) Gillespie, D. T. J. Phys. Chem. 1977, 81, 2340.

(53) Gillespie, D. T. Annu. Rev. Phys. Chem. 2007, 58, 35.

(54) Al-Harthi, M.; Khan, M. J.; Abbasi, S. H.; Soares, J. B. P. Macromol. React. Eng. 2009, 3,148 .

(55) Coropceanu, V.; Cornil, J.; da Silva, D. A.; Olivier, Y.; Silbey, R.; Bredas, J. L. Chem. Rev. 2007, 107, 926.

(56) Tessler, N.; Preezant, Y.; Rappaport, N.; Roichman, Y. Adv. Mater. 2009, 21, 2741.

(57) Shuai, Z.; Geng, H.; Xu, W.; Liao, Y.; Andre, J.-M. Chem. Soc. Rev. 2014, 43, 2662.

(58) Auer, S.; Frenkel, D. Nature 2001, 409, 1020.

(59) Auer, S.; Frenkel, D. J. Chem. Phys. 2004, 120, 3015.

(60) Wang, Z. Y.; Li, Y. H.; Adams, J. B. Surf. Sci. 2000, 450, 51.

(61) Piana, S.; Reyhani, M.; Gale, J. D. Nature 2005, 438, 70.

(62) Piana, S.; Gale, J. D. J. Cryst. Growth 2006, 294, 46.

(63) Toth, G. Cryst. Growth Des. 2008, 8, 3959. 
(64) Reilly, A. M.; Briesen, H. J. Cryst. Growth 2012, 354, 34.

(65) Girit, C. O.; Meyer, J. C.; Erni, R.; Rossell, M. D.; Kisielowski, C.; Yang, L.; Park, C. H.; Crommie, M. F.; Cohen, M. L.; Louie, S. G.; Zettl, A. Science 2009, 323, 1705.

(66) Kotakoski, J.; Jin, C. H.; Lehtinen, O.; Suenaga, K.; Krasheninnikov, A. V. Phys. Rev. B 2010, 82, 113404.

(67) Nie, Y. F.; Liang, C. P.; Zhang, K. H.; Zhao, R.; Eichfeld, S. M.; Cha, P. R.; Colombo, L.; Robinson, J. A.; Wallace, R. M.; Cho, K. 2D Mater. 2016, 3, 025029.

(68) Nie, Y.; Liang, C.; Cha, P.-R.; Colombo, L.; Wallace, R. M.; Cho, K. Sci. Rep. 2017, 7, 2977.

(69) Koo, B. T.; Heden, R. F.; Clancy, P. Phys. Chem. Chem. Phys. 2017, 19, 9745.

(70) Al-Harthi, M. A.; Masihullah, J. K.; Abbasi, S. H.; Soares, J. B. P. Macromol. Theory Simul. 2009, 18, 307.

(71) Hamzehlou, S.; Reyes, Y.; Leiza, J. R. Macromol. React. Eng. 2012, 6, 319.

(72) Hamzehlou, S.; Reyes, Y.; Leiza, J. R. Macromolecules 2013, 46, 9064.

(73) Vekilov, P. G. Cryst. Growth Des. 2010, 10, 5007.

(74) Palberg, T. J. Phys.-Condes. Matter 2014, 26, 333101.

(75) Chavez, A. D.; Smith, B. J.; Smith, M. K.; Beaucage, P. A.; Northrop, B. H.; Dichtel, W. R. Chem. Mater. 2016, 28, 4884.

(76) Sun, S. F. Physical Chemistry of Macromolecules: Basic Principles and Issues; John Wiley \& Sons, Inc.: Hoboken, 2004.

(77) Vekilov, P. G. Cryst. Growth Des. 2004, 4, 671.

(78) Cölfen, H.; Antonietti, M. Mesocrystals and Nonclassical Crystallization; John Wiley \& Sons, Ltd.: Chichester, 2008.

(79) Zhang, Q.; Liu, S. J.; Yu, S. H. J. Mater. Chem. 2009, 19, 191.

(80) Vekilov, P. G. Nanoscale 2010, 2, 2346.

(81) De Yoreo, J. Nat. Mater. 2013, 12, 284.

(82) Ivanov, V. K.; Fedorov, P. P.; Baranchikov, A. Y.; Osiko, V. V. Russ. Chem. Rev. 2014, $83,1204$.

(83) tenWolde, P. R.; Frenkel, D. Science 1997, 277, 1975.

(84) Yau, S. T.; Vekilov, P. G. Nature 2000, 406, 494.

(85) Oxtoby, D. W. Nature 2000, 406, 464.

(86) Yau, S. T.; Vekilov, P. G. J. Am. Chem. Soc. 2001, 123, 1080.

(87) Schilling, T.; Schope, H. J.; Oettel, M.; Opletal, G.; Snook, I. Phys. Rev. Lett. 2010, 105, 025701.

(88) Banfield, J. F.; Welch, S. A.; Zhang, H. Z.; Ebert, T. T.; Penn, R. L. Science 2000, 289, 751. 


1
2
3
4
5
6
7
8
9
10
11
12
13
14
15
16
17
18
19
20
21
22
23
24
25
26
27
28
29
30
31
32
33
34
35
36
37
38
39
40
41
42
43
40
45
49
50
51
52
53
55
50

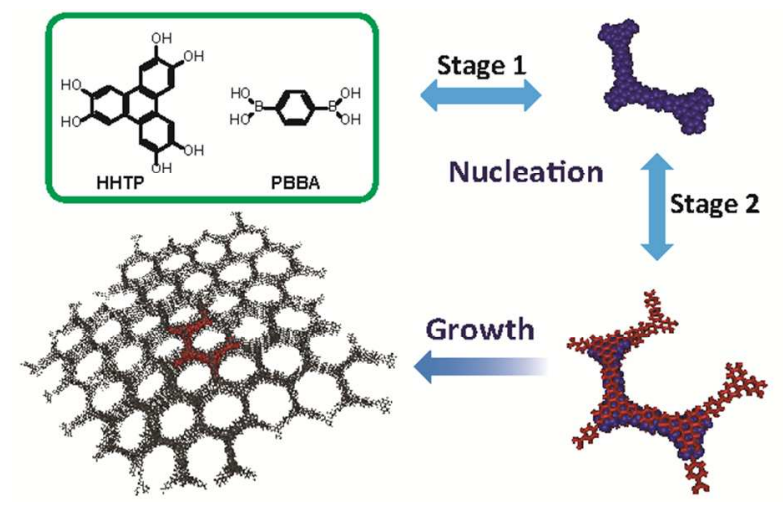

16

17

18

19

20

22

23

24

26

27

28

29

32

33

34

35

36

39

40

41

42

43

44

46

47

48

49

50

52

53

54

55

58

59

60 